\title{
1.45 Tbit/s, Low Latency Data Transmission through a 19-Cell Hollow Core Photonic Band Gap Fibre
}

\author{
R. Slavík, M.N. Petrovich, N.V. Wheeler, J.R. Hayes, N.K. Baddela, D. Gray, F. Poletti, and \\ D.J.Richardson \\ Optoelectronics Research Centre, University of Southampton, Highfield Campus, SO17 1BJ, \\ Southampton, UK, r.slavik@soton.ac.uk
}

\begin{abstract}
We report transmission of $37 \times 40$ Gbit/s C-band channels over $250 \mathrm{~m}$ of hollow core band gap fibre, at $99.7 \%$ the speed of light in vacuum. BER penalty below $1 \mathrm{~dB}$ as compared to back-toback was measured across the $C$-band.
\end{abstract}

\section{Introduction}

Hollow core-photonic bandgap fibres (HCPBGFs) have many intriguing properties including ultralow nonlinearity and low-latency relative to conventional (solid) forms of optical fibre. As a consequence they are of great interest as a potential transmission medium in next-generation optical communication systems. However, in order to have any chance of competing with conventional transmission fibres low loss over a suitably extended bandwidth needs to be achieved. Since loss in such fibres is ultimately limited by scattering at the silica/air core interface, ultralow loss will only ever be achieved by moving to large core designs that are inherently multi-mode. Consequently, it is evident that the ability to reliably control and manage modal content within such fibres will be a further fundamental requirement if such fibres are ever to be used in optical fibre communications.

Recently, we have reported ${ }^{1[1]}$ for the first time a HC-PBGF which simultaneously provides for low loss $(3.5 \mathrm{~dB} / \mathrm{km})$ and a wide transmission bandwidth (160 nm 3-dB bandwidth). This was achieved by combining a 19-cell core design, promising low loss ${ }^{[2] 2}$, with a thin wall surround ${ }^{3}$ that enables elimination of surface modes from the central part of the band gap. We also showed that although the HC-PBGF was indeed multimode, it could be operated in an effectively single-mode regime. This was achieved by buttcoupling it to two SMF-28 fibres, providing selective input and output coupling into the $\mathrm{LP}_{01}$ mode. However, to convincingly prove that the fibre can be used to transmit data with negligible penalty due to modal interference requires a transmission system experiment. Note that to the best of our knowledge the highest reported data transmission capacity in a HC-PBGF is $10 \mathrm{Gbit} / \mathrm{s}$ achieved in single-channel experiments over $150 \mathrm{~m}$ of significantly less multimode 7 -cell core fibre ${ }^{4}$.
Herein we report the results of transmission experiments in our new 19-cell HC-PBGF. Due to lack of surface modes across the C-band and the low intermodal cross-talk we successfully demonstrate low-latency transmission of $37 \mathrm{x}$ 40 Gbit/s C-band DWDM channels over $250 \mathrm{~m}$ of fibre, which equates to a total transmission capacity of almost $1.5 \mathrm{Tbit} / \mathrm{s}$. Whilst it will clearly ultimately be necessary to demonstrate transmission over far greater lengths in order to establish the viability of using HC-PBGFs in long-haul optical fibre communications, lowlatency transmission over $<\mathrm{km}$ scale distances is already of potential interest in certain data centre/distributed computing applications.

\section{Fabrication}

The 19-cell HC-PBGF was fabricated using the conventional stack and draw technique. Through

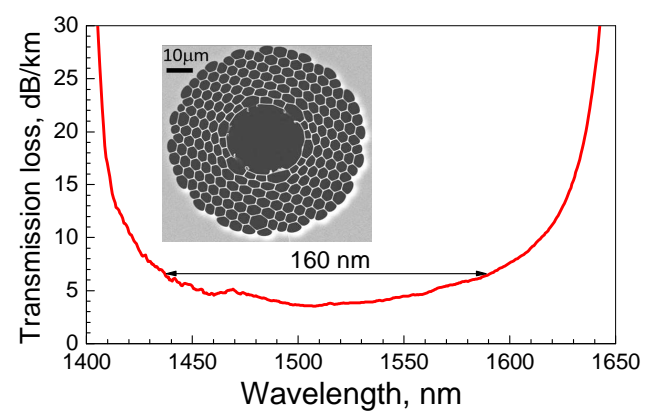

Fig. 1: Cut-back loss measurement of the used HC-PBGF; SEM in the inset.

careful control of draw parameters it was possible to push the spectral location of residual surface modes towards the edges of the band gap. The fabricated fibre has $61 / 2$ rings of cladding holes with an average pitch and relative hole size of $4.4 \mu \mathrm{m}$ and 0.97 respectively, and a core size of $26 \mu \mathrm{m}$ (Fig. 1, inset). Its transmission loss, measured over a $250 \mathrm{~m}$ fibre sample, is shown in Fig. 1. A minimum transmission loss of $3.5 \mathrm{~dB} / \mathrm{km}$ at $\sim 1500 \mathrm{~nm}$ and a very wide surface mode free 
central region with $3 \mathrm{~dB}$ transmission bandwidth of $160 \mathrm{~nm}$ were achieved. As is well known, scaling the central wavelength of the fibre to $\sim 2 \mu \mathrm{m}$ should further reduce the loss relative to | a fibre designed for $1.55 \mu \mathrm{m}$ operation ${ }^{[5] 5}$.

\section{Single-mode operation}

First, we used a time-of-flight (TOF) method to evaluate how well the HC-PBGF could be operated in a single-mode regime. We used 1020 ps pulses emitted at $20 \mathrm{MHz}$ repetition rate by a passive mode-locked fibre laser and detected them using a $10-\mathrm{GHz}$ bandwidth photodiode and fast sampling oscilloscope. We used butt-coupling with SMF-28 fibre to couple light into and out of the fibre. The total fibre loss including the two butt-coupled connections was measured to be below $6 \mathrm{~dB}$. The losses are mainly due to the mismatch of the mode field diameter of SMF-28 (about $8 \mu \mathrm{m}$ ) and that of the fundamental mode of the HC-PBGF (about $16 \mu \mathrm{m})$. Thus, we expect that lower coupling loss may be achieved using a fibre launch with better matched mode field diameter. The results of TOF measurement made at a signal wavelength of $1556 \mathrm{~nm}$ for optimum coupling at the input and output of the HC-PBGF are shown in Fig. 2. By 'optimum coupling' we mean minimum loss for the fundamental mode with

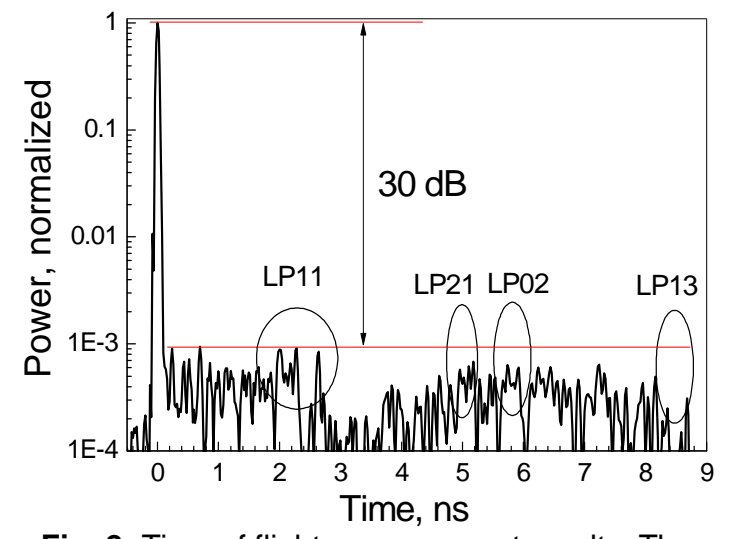

Fig. 2: Time of flight measurement results. The positions of higher order modes, measured in an earlier experiment ${ }^{1}$, are also shown.

simultaneous maximum suppression of higher order modes. For better visualization of the noise and contribution from higher order modes, a log scale is used in Fig. 2. The (measured) response of the fast photodiode used exhibited some ringing in the $0-2.5$ ns range at the $-22 \mathrm{~dB}$ level which we have subtracted in the data shown in Fig. 2. Here, we see that higher order modes are suppressed by more than $30 \mathrm{~dB}$ as compared to the fundamental mode. In Fig. 2, the positions of higher order modes that were measured with an offset launch into HC-PBGF earlier $^{1}$ are also marked. We see that any contribution from these modes is buried in the noise floor of our measurement which has a dynamic range of more than $30 \mathrm{~dB}$. This is a clear demonstration that our HC-PBGF can be efficiently operated as 'single-mode' transmission medium.

\section{Transmission experiment}

Our transmission experiment set-up is shown in Fig. 3. First, we combined 37 lasers emitting on a $100-\mathrm{GHz}$ ITU grid over almost the entire Cband (1528-1560 nm) - the spectrum of the

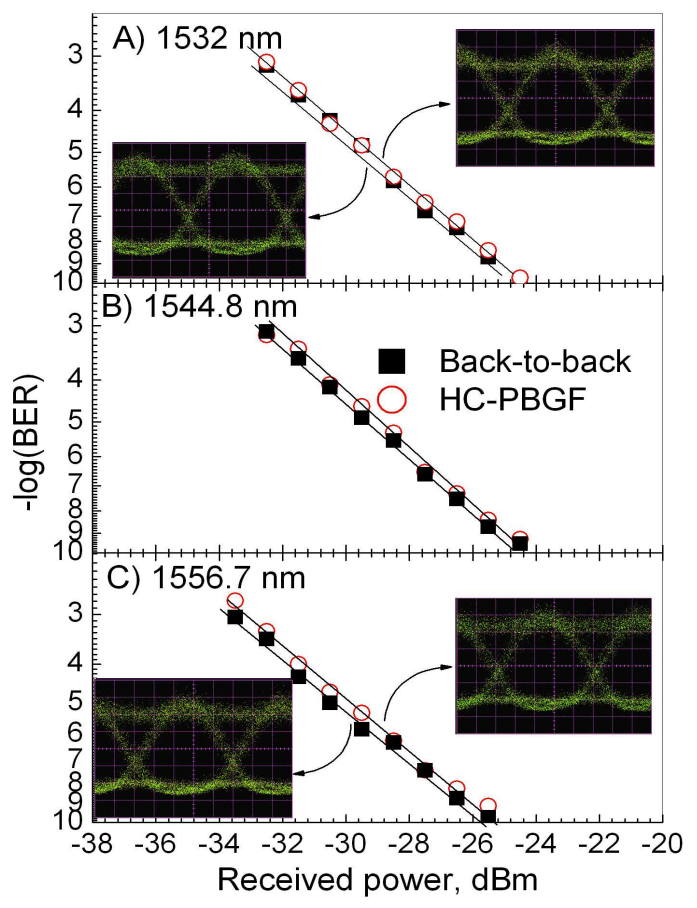

Fig. 4: Back-to-back and HC-PBGF at three selected channels. The eye diagrams show almost negligible degradation due to propagation through the HC-PBGF.

combined lasers obtained at $0.1 \mathrm{~nm}$ resolution is shown in Fig. 3. Here we see that three ITU channels are not occupied due to earlier failure of these devices. Subsequently, all lasers were
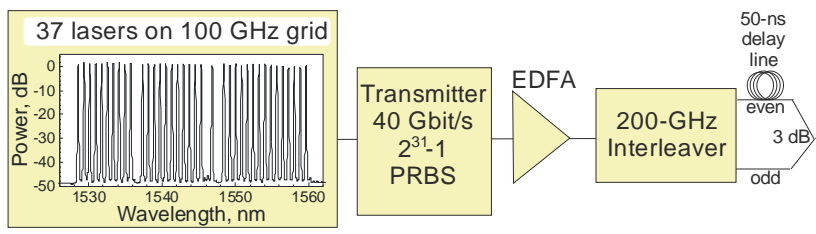
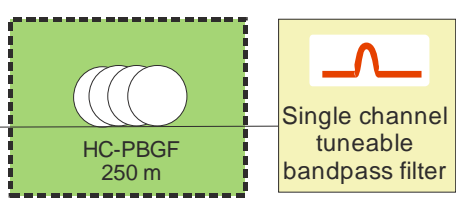

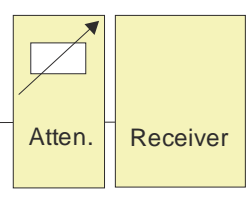

Fig. 3: Set-up of the transmission experiment. 
modulated using a Mach-Zehnder amplitude modulator with $40 \mathrm{Gbit} / \mathrm{s}$ OOK data with a $2^{31}-1$ pseudorandom bit sequence (PRBS). To decorrelate the neighboring channels, we split the odd and even number channels in an interleaver (a 2-channel programmable wavelength selective switch) and delayed the even-number channels by $50 \mathrm{~ns}$ (10 m of SMF-28) with respect to the odd channels. After recombination of the odd and even channels in a 3-dB coupler, the data was launched into a 250$\mathrm{m}$ sample of HC-PBGF via butt coupling. The measured loss of this particular sample is shown in Fig. 1. At the HC-PBGF output, the signal was butt coupled back into SMF-28, one data channel at a time was filtered via a band-pass filter and analyzed using the optically preamplified receiver. First, we compared the Bit Error Ratio (BER) when propagating data through the HC-PBGF with back-to-back measurements (for which the HC-PBGF was removed) for several channels. Results for three representative channels - two towards the edges of the C-band and one in its middle are shown in Fig. 4. We see that in all the cases the power penalty is almost negligible. This is also confirmed by observing the corresponding eye diagrams shown as insets in Fig. 4. We believe that the very slight power penalty observed may be caused by the reflection at the butt-coupled SMF-28 to HC-PBGF interfaces rather than by inter-modal coupling inside the HC-PBGF.

The comparison of performance in terms of BER of several channels over the entire spectral bandwidth used is shown in Fig. 5. Here we see that the difference in BER sensitivity is less than $1.5 \mathrm{~dB}$ across all the measured channels, similar to the back-to-back case (not shown).

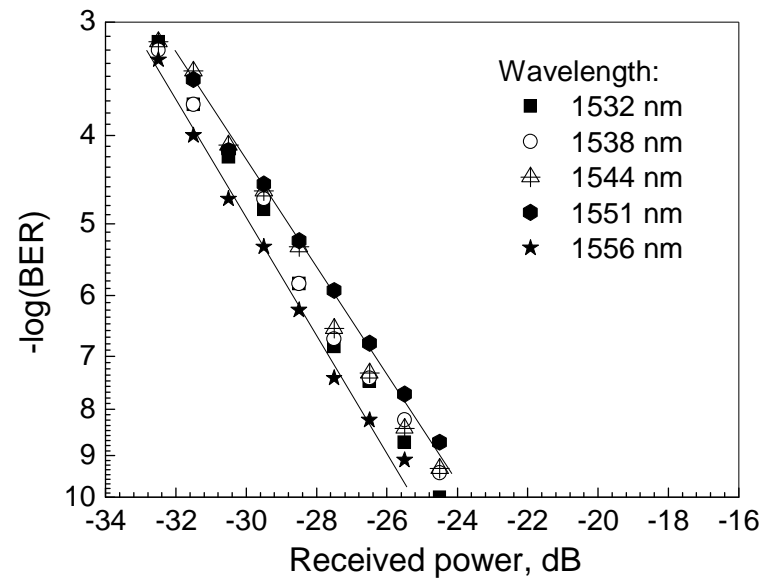

Fig. 5: HC-PBGF BER curves for 5 selected channels across the transmission band.

\section{Discussion and conclusions}

The data transmission capability of recentlyfabricated 19-cell HC-PBGF with $3.5 \mathrm{~dB} / \mathrm{km}$ loss and 3-dB transmission band exceeding $160 \mathrm{~nm}^{1}$ for high capacity low-latency single-mode transmission was experimentally studied. Using mode-selective coupling we effectively achieved single-mode operation for a $250 \mathrm{~m}$ long fibre sample. The modal guidance properties were characterized using a Time of Flight Method, which showed better than $30 \mathrm{~dB}$ suppression of higher-order modes. Subsequently, we launched 37 DWDM channels placed on a $100-\mathrm{GHz}$ ITU grid, each carrying $40 \mathrm{Gbit} / \mathrm{s}$ On-Off keyed data streams, and measured the BER transmission penalty. For all measured channels the BER penalty (as compared to the back-to-back case) was well below $1 \mathrm{~dB}$.

Besides its extremely low nonlinearity, another attractive feature of this fibre is its lowlatency, as the light propagates 1.45 times faster than in a standard solid-core fibre. This is believed to be of potential interest in a number of short reach applications, e.g., to remove bottlenecks in high performance supercomputers, or to promote ultra-fast interconnections in data centres.

It is worth mentioning that by extrapolating these loss results with an accurate scattering loss model, we believe there is a realistic prospect of further reducing losses in these wide bandwidth HC-PBGFs down to the 0.2-0.4 $\mathrm{dB} / \mathrm{km}$ level at $2 \mu \mathrm{m}$ wavelengths, potentially opening up the possibility for longer transmission distance applications.

\section{Acknowledgements}

This work was supported by the EU 7th Framework Programme under grant agreement 228033 (MODE-GAP) and by UK EPSRC grants EP/I01196X/1 and EP/H02607X/1.

\section{References}

[1] N. Wheeler et al, "Wide-bandwidth low-loss 19-cell hollow core photonic band gap fiber and its potential for low latency data transmission," PDP5A.2, OFC 2012.

[2] B. J. Mangan, et al., "Low loss (1.7 dB/km) hollow core photonic bandgap fiber," PDP 24, OFC 2004.

[3] R. Amezcua-Correa, et al., "Design of 7 and 19 cells core air-guiding photonic crystal fibers for low-loss, wide bandwidth and dispersion controlled operation," Optics Express 15, 17577-17586 (2007).

[4] C. Peucheret, B. Zsigri, T.P. Hansen, and P. Jeppesen, "10 Gbit/s transmission over airguiding photonic bandgap fiibre at $1550 \mathrm{~nm}$," Electron. Lett. 41 (2004).

[5] P.J. Roberts, et al., "Ultimate low loss of hollow-core photonic crystal fibres," Optics Express 13, 236-244 (2005). 